\title{
A laboratory based system for Laue micro x-ray diffraction
}

\author{
P. A. Lynch, ${ }^{\text {a }}$ A. W. Stevenson, D. Liang, D. Parry, and S. Wilkins \\ Commonwealth Scientific and Industrial Research Organization, Manufacturing \\ and Infrastructure Technology, Private Bag 33, Clayton South MDC, 3169, Australia \\ N. Tamura \\ Advanced Light Source, Lawrence Berkeley National Laboratory, 1 Cyclotron Road, \\ Berkeley, California 94720
}

\begin{abstract}
A laboratory diffraction system capable of illuminating individual grains in a polycrystalline matrix is described. Using a microfocus $\mathrm{x}$-ray source equipped with a tungsten anode and prefigured monocapillary optic, a micro-X-ray diffraction system with a $10 \mathrm{~m}$ beam was developed. The beam profile generated by the ellipsoidal capillary was determined using the "knife edge" approach. Measurement of the capillary performance, indicated a beam divergence of $14 \mathrm{mrad}$ and a useable energy bandpass from 5.5 to $19 \mathrm{keV}$. Utilizing the polychromatic nature of the incident $\mathrm{x}$-ray beam and application of the Laue indexing software package X-Ray Micro-Diffraction Analysis Software, the orientation and deviatoric strain of single grains in a polycrystalline material can be studied. To highlight the system potential the grain orientation and strain distribution of individual grains in a polycrystalline magnesium alloy $\mathrm{Mg} 0.2 \mathrm{wt} \%$ $\mathrm{Nd}$ was mapped before and after tensile loading. A basal 0002 orientation was identified in the as-rolled annealed alloy; after tensile loading some grains were observed to undergo an orientation change of $30^{\circ}$ with respect to 0002 . The applied uniaxial load was measured as an increase in the deviatoric tensile strain parallel to the load axis.
\end{abstract}

\section{INTRODUCTION}

Historically, the potential of the Laue method has been restricted to single crystal and large grain crystal structures. Such restrictions are the result of limited flux in laboratory x-ray sources and the complex nature of experimental Laue patterns arising from polycrystalline materials. Using the continuous spectrum of high brilliance synchrotron sources, precision focusing optics, along with the availability of high speed computers, the potential of the Laue method has been rediscovered in recent years. For example, Laue micro-X-ray diffraction XRD has been used to study the orientation, ${ }^{1}$ residual stress, ${ }^{2}$ and defect concentration ${ }^{3,4}$ within individual grains of polycrystalline materials. With focused spot sizes less than $100 \mathrm{~nm}$ reported, 5 XRD shows potential for solv- ing problems presently encountered in existing microscopy techniques. For example, TEM studies of heavily deformed $\mathrm{Mg}$ alloys are problematic due to contrast overlap caused by large concentrations of dislocations and twinning defects. Similar problems have also been observed in electron backscattering diffraction studies. ${ }^{6}$

Although synchrotron beamlines lead the development of future XRD experimentation, an inherent problem which is particularly apparent in this field is the increasing demand and limited availability of synchrotron-based XRD re- sources. Utilizing modern $\mathrm{x}$-ray source and optics develop- ment, laboratory XRD instrumentation and experimenta- tion is an area of growing interest. In recent years, the

aElectronic mail: Peter.Lynch@csiro.au successful application of laboratory XRD instrumentation has led to the availability of commercial systems with a spatial resolution ranging from 50 to $300 \mathrm{~m} .^{7}$

Microbeam formation processes rely on the ability to efficiently image or demagnify the $x$-ray source onto the sample. Various formation techniques have been proposed and one of the most successful methods, based on mirror focusing, was pioneered in the 1950s. ${ }^{8}$ More recently, other focusing mechanisms have been studied by many research- ers. Some of these optic designs include zone plates, ${ }^{9,10}$ single/poly capillaries, ${ }^{11}$ microchannel plates, ${ }^{12}$ and compound refractive lenses. ${ }^{13}$ Although many focusing optics ex- ist, each approach is intrinsically linked to the properties of the $\mathrm{x}$ ray source and focus requirements, consequently most focusing methods are application specific. ${ }^{14}$ For example, white beam XRD requires a focusing optic with high spa- tial resolution and broad energy bandpass; Kirkpatrick-Baez mirrors and capillary optics have traditionally been used to meet these demands. ${ }^{11}$

Capillary focusing is one area of $\mathrm{x}$-ray optics research that has undergone considerable development, especially for the generation of microbeams using laboratory $\mathrm{x}$-ray sources. Several competing factors must be satisfied to achieve an optimal capillary design. These include the focused beam size requirements, beam divergence, and $\mathrm{x}$-ray source. ${ }^{15}$ The physical basis underpinning capillary design is the total ex- ternal reflection of $\mathrm{x}$ rays from a smooth surface. ${ }^{16}$ Conse- quently material and surface roughness properties play a leading role in describing the optic performance. Typically glass capillary optics are preferred due to their high / 


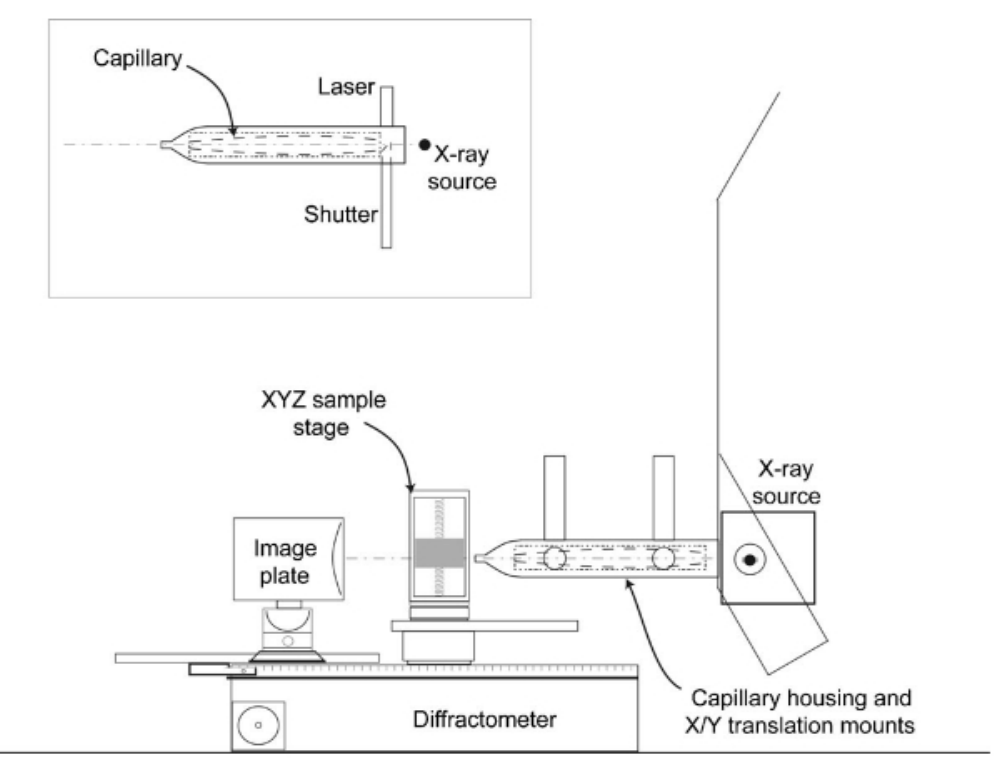

FIG. 1. Schematic illustration showing the design and geometry of the laboratory XRD system. The inset provides a detailed view of the capillary housing and shutter/mirror system with respect to the laser used for sample alignment.

ratio, where represents the imaginary absorption component and the real dispersion component of the complex refractive index. One of the first demonstrations of fiber technology as applied to capillary optics was reported by Rindby $1986,{ }^{17}$ the relationship between $\mathrm{x}$-ray energy and observed $\mathrm{x}$-ray deviation in a pyrex glass fiber was quantified by measurement of the critical angle for total reflection. Using a straight capillary design, beam sizes down to about $10 \mathrm{~m}$ were achieved from standard x-ray tubes. ${ }^{18}$

Subsequent optic design has utilized drawing processes to manufacture prefigured geometries that exhibit superior performance characteristics. Some of these shapes include; tapered/conical, ${ }^{19,20}$ parabolic, $^{21}$ and ellipsoidal. ${ }^{22}$ A common feature of these prefigured optics is their condensing type nature, i.e., photons entering the capillary bore undergo total external reflection along the optic length until they emerge from the exit bore. This can be problematic because the inner wall surface roughness plays a major role in terms of the transmission efficiency. Moreover, a reduction in the beam size introduces increased beam divergence which places sig- nificant restrictions on the sample positioning to achieve maximum flux from the optic. It is found that the transmis- sion efficiency of capillary devices can be drastically im- proved by reducing the number of reflections a photon must undergo. ${ }^{23}$ Improvements in this area have been reported through the development of single bounce optics; it has been demonstrated that a paraboloidal prefigured capillary is ca- pable of producing a transmission efficiency of $90 \%$ with the focal plane well beyond the exit aperture. ${ }^{15}$ Most re- cently, it has been highlighted that as a result of the improve- ments initiated by prefigured optics, the major gain is pres- ently achieved by minimizing the slope deviation from the ideal guiding figure. ${ }^{14}$

Quality prefigured optics coupled with high brilliance microfocus X-ray sources have displayed considerable prom- ise in laboratory applications. In general, monocapillary and polycapillary optics are used for diffraction and fluorescence measurements, respectively. Using a parabolic optic and cus- tom built $\mathrm{x}$-ray generator Yamamoto 24 designed a laboratory instrument with a spatial resolution of $0.8 \mathrm{~m}$. X-ray fluo- rescence XRF and diffraction spectra were used to study the microstructural properties of high performance ultralarge-scale integrated devices. Using a laboratory XRF spectrometer equipped with a capillary optic, Wegrzynek ${ }^{25}$ conducted a feasibility study highlighting the capabilities of absorption based computer microtomography imaging. Case studies were carried out on various samples and a spatial resolution of a few tens of micrometers was reported. Based on a modular design, Bjeoumikhov et al. ${ }^{26}$ used a commer- cial low-power microfocus source with various polycapillary optics to perform XRF and XRD. For the diffraction ex-periments absorbing foils were introduced in order to obtain a reasonable degree of monochromaticity in the primary beam. One of the underlying features apparent in the laboratory-based $\mathrm{XRD}$ literature is that although the studies highlighted the natural polychromatic bandpass of the capil- lary, diffraction experiments were confined to the high-

intensity characteristic lines of the incident beam/source.

In the present contribution a laboratory system that utilizes the background continuum radiation to collect polychromatic Laue data is reviewed. Measurements have been performed to address the properties of the incident beam pro- file and performance of the capillary optic. Finally, applica- tion of the laboratory XRD system to study the individual grain orientation and deviatoric strain in a polycrystalline magnesium alloy $\mathrm{MgNd}$ magnesium $99.98 \%$, $\mathrm{Nd} 0.2 \%$ is demonstrated.

\section{MICRODIFFRACTION SYSTEM}

The x-ray microdiffraction system developed in this work is illustrated in Fig. 1. The specific microstructural information that can be studied with this instrumentation is highly sample dependent, in particular, grain size dependent. In the first instance, where the grain size is less than the focused $\mathrm{x}$-ray spot size, crystal structure information relating to the phase, grain domain size, and macroscopic deforma- tion stress/strain relationships can be achieved. Alternately, when the sample grain size is of similar dimensions or greater than the incident $\mathrm{x}$-ray beam, grain specific informa- 
tion can be realized. Accordingly, the instrumentation design has been optimized for the latter case, i.e., Laue XRD stud- ies. As illustrated in Fig. 1, a laboratory based XRD system requires several key hardware components, including; a mi- crofocus $\mathrm{X}$ ray source, focusing capillary x-ray optic, high-

resolution sample mapping stage and area detection system.

The objective in designing the source and preoptic configuration illustrated in Fig. 1 was to maximize the number of photons incident upon the sample within the desired spot size without introducing excessive beam divergence. Accord- ingly, it was possible to produce a $10 \mathrm{~m}$ x-ray spot by using a commercially available microfocus x-ray source Feinfo- cus equipped with a $\mathrm{W}$ anode and a focusing capillary $\mathrm{x}$-ray optic AXCO . Based on the resources available for calculat- ing the efficiency of a $10 \mathrm{~m}$ focal spot for XRD applica- tions, the underpinning system features were the source

brightness and capillary gain/efficiency.

For the present application, the x-ray source figure of merit is brightness which is directly related to the maximum power density, and therefore the minimum beam diameter of the incident electron beam which can be delivered to the target anode without causing damage. Using the steady state heat flow equation, Grider et al. ${ }^{27}$ presented a method for measuring the electron beam radius and hence $x$-ray source radius in a microfocus tube as a function of the electron beam power corresponding to the onset of target damage. For three different target materials, a linear dependence was ob- served between the source size and applied power. As an approximate guide, the source size scaled according to the applied power in a 1:1 ratio, i.e., for each watt of power the source size increases by 1 $\mathrm{m}^{2}$. For the present microfocus $\mathrm{x}$-ray source, based on the manufacturer's guidelines, the op- erating conditions range from 0-160 kV with an anode cur- rent of 0-3 mA. Experiments were undertaken to determine the optimum source operating conditions. Although depen- dent upon the efficiency of the capillary optic and x-ray source power limits, the experimental settings used in this study were $30 \mathrm{kV}$ and 1-3 $\mathrm{mA}$, a maximum power of $90 \mathrm{~W}$, with a corresponding source size ranging from 30 to $90 \mathrm{~m}^{2}$.

A focusing capillary optic supplied by $\mathrm{AXCO}^{28}$ was manufactured according to the x-ray source size, focus properties, and spectral transmission requirements. To optimize the capillary gain and efficiency, the ability to model the transmission efficiency according to profile shape ${ }^{23}$ and to accurately fabricate a predefined capillary profile was essen- tial; this task was conducted by AXCO. Using their novel fabrication technique an ellipsoidal shaped optic $110 \mathrm{~mm}$ long, with a theoretical focal spot size of $10 \mathrm{~m}$ and gain of 180 was manufactured for the XRD system.

The inset of Fig. 1 shows a schematic illustration of the capillary design with reference to the source position. Sev- eral key manufacturing features were essential for this de- sign, including location of the capillary entrance aperture $12 \mathrm{~mm}$ away from the source, this enabled the standard Be win- dow on the source to remain in place. The optic was also fixed within two brass cylinders, the smaller diameter cylin- der positioning the optic while the larger diameter provides mechanical strength and support for Oriel DC Mike motors needed to automate $X$ and $Y$ translations near the entry and exit bore of the capillary. Moreover, this design acts as a form of shielding, minimizing background leakage caused by high energy x rays penetrating through the capillary walls. At the capillary focal plane an aperture was added to restrict the component of the direct beam passing through the capillary without undergoing reflection. Although the intensity of this component of the direct beam is small compared to the focused spot, the aperture was justified as it reduces the inten- sity in the tail of the incident spot distribution. ${ }^{15}$ Further- more, the aperture also blocks incident $\mathrm{x}$ rays that may have undergone transmission through the capillary walls.

To accurately align the sample and to perform localized area mapping studies, a high-resolution $X Y Z$ stage was mounted on the diffractometer axis of rotation. Positioning the specimen region of interest with respect to the x-ray fo- cus was achieved by the introduction of a laser beam onto the sample surface along a coaxis of the x-ray beam, as shown in Fig. 1. To collect the diffraction data high- resolution image plates $200 \mathrm{~mm} 250 \mathrm{~mm}, 25 \mathrm{~m}$ pixel size were positioned on a slide rail mounted on the 2 arm of the diffractometer. For indexation purposes it was essen- tial to provide an accurate and reproducible method for po- sitioning the image plate with respect to the incident beam. This aspect was resolved by manufacturing an image plate holder which incorporated a series of apertures for precise relocation after processing. Moreover, due to the physical size of the image plate it was possible to record the position of the direct beam which was also used as a further reference point.

\section{A. Characteristics of the incident x-ray beam 1. Optic alignment}

Capillary alignment was automated by Oriel $X$ and $Y$ translation drives mounted perpendicularly to the beam propagation direction at the entrance and exit of the capillary. Observation of the beam issuing from the capillary was achieved by imaging the focused spot using a charge-coupled device CCD detector placed approximately $50 \mathrm{~mm}$ from the focal plane. When the capillary was suitably aligned an intense spot surrounded by an annulus of comparable inten- sity was observed Fig. 2 . The annulus could be moved with small translations of the capillary tip, however the central spot remained stationary during these translations. From these observations it was concluded that the central spot con- sisted of $\mathrm{x}$-rays that had not been reflected by the capillary walls and was emanating directly from the source. The sur- rounding annulus corresponded to $\mathrm{x}$ rays undergoing total external reflection from the capillary walls, diverging past the focal plane.

In an attempt to provide a quantitative method for the optic alignment a scintillation detector with a pulse height discriminator set at various energy levels $\mathrm{Cu} K$, Mo $K$ was used to measure the observed count rate as a function of the capillary translation position. Although this approach provided a measure of the incident intensity, this was problematic because it was necessary to attenuate the direct beam with Al filters, resulting in beam hardening. Bearing this in 


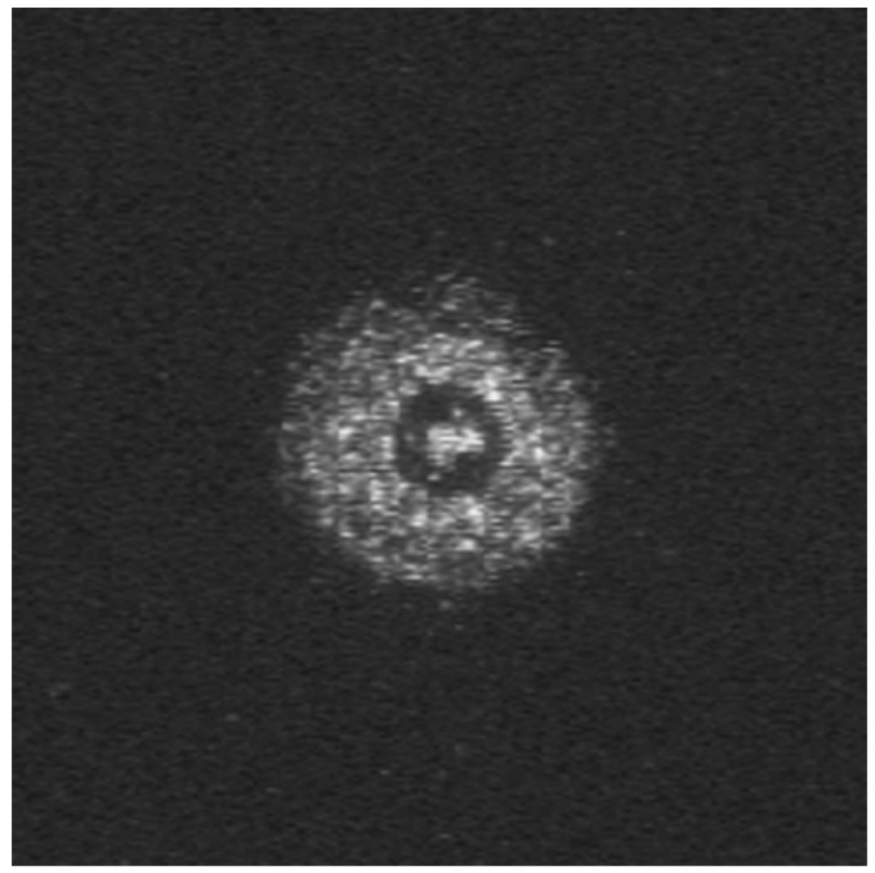

FIG. 2. CCD image recorded at a distance of $50 \mathrm{~mm}$ from the end of the exit aperture of the optic, showing the central spot surrounded by a uniform annular ring due to x-rays undergoing total external reflection from the capillary walls.

mind it was possible to minimize this effect by recording the output energy spectrum of the incident beam using an energy dispersive Si Li detector.

\section{X-ray focus}

Once the $x$-ray throughput of the optic was maximized, the size and profile of the emerging beam was determined using the knife edge method. ${ }^{19}$ To perform this task a piece of tantalum with a highly polished edge was mounted paral-

lel to the face of the aperture on the high-resolution $X Y Z$ translation stage. A scintillation detector set for $\mathrm{Cu} K$ and Mo $K$, positioned $50 \mathrm{~mm}$ from the aperture, was used to monitor the observed incident beam intensity as the knife edge was translated laterally through the beam issuing from the capillary. Stepping in $1.0 \mathrm{~m}$ increments enabled one to estimate the beam size in the horizontal and vertical direc- tions, Fig. 3 a highlights this result for translation of the knife edge in the horizontal $X$ direction. The rate of change of the observed intensity with respect to the knife edge po- sition was calculated and the full width at half maximum

FWHM intensity found. Figure $3 \mathrm{~b}$ shows this differential

data and the calculated FWHM obtained from fitting a Gaussian function to the data; in this instance the fitted FWHM was $9.2 \mathrm{~m}$. In performing subsequent analysis, the fitted Gaussian function of the differential data was highly dependent on the initial and final slope of the knife edge data, illustrated in Fig. 3 a , corresponding to no interception of and then totally blocking the beam, respectively. As a result the FWHM could only be used as an approximate measure of the beam size emanating from the capillary. In- dependent of this discrepancy, the result confirms that the beam was confined within the capillary bore and exit defin- ing aperture. Furthermore, this confirms the present source/ optic configuration produces a $10 \mathrm{~m}$ incident beam.
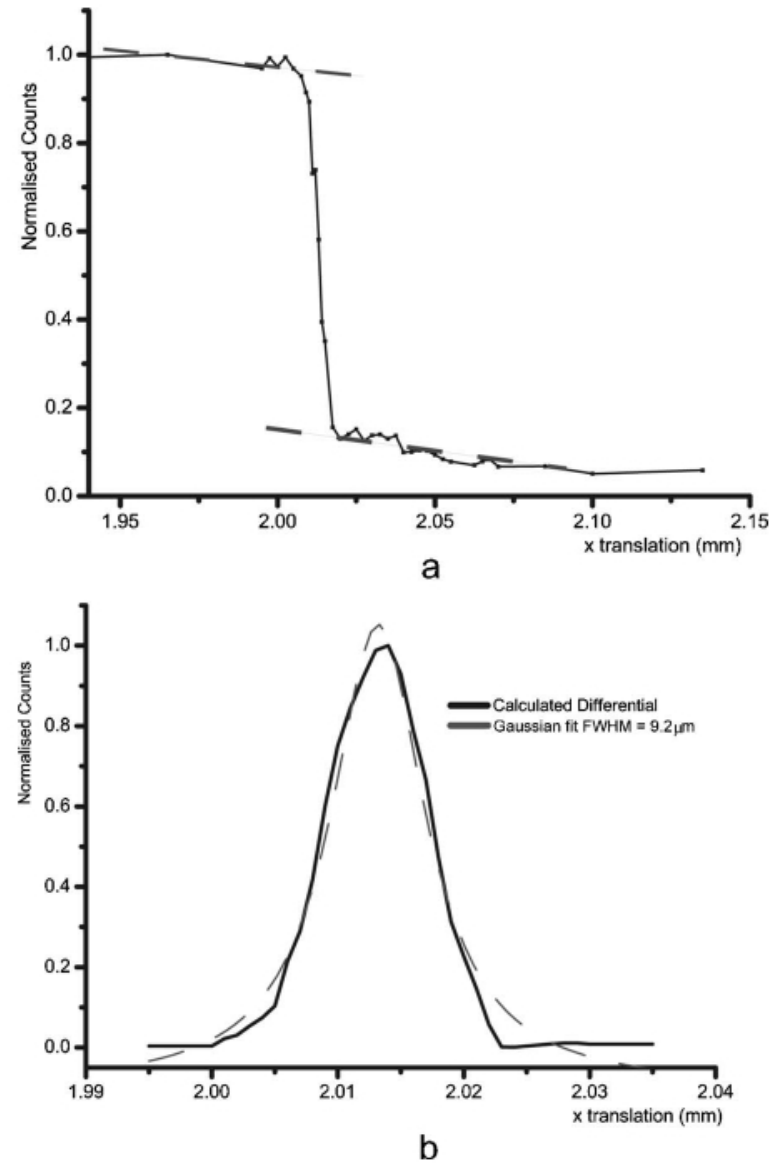

FIG. 3. Knife edge test of the beam emerging from the capillary/aperture. Data were collected using a scintillation detector with the pulse height discriminator set at $8.04 \mathrm{keV}$. a Data collected as the knife edge undergoes $\mathrm{x}$ translation. b Differential data of the knife edge experiment and corresponding Gaussian fit with a FWHM= $9.2 \mathrm{~m}$.

\section{Beam divergence and optic gain}

Two parameters intrinsically linked to the performance of a focusing capillary are the beam divergence and optic gain. In other words, the increased x-ray flux at the focal plane achieved with the optic must be considered in terms of the level of collimation necessary to satisfy the experimental requirements. For example, an area of intended research that will be undertaken using the present instrumentation is the analysis of smearing in Laue patterns arising from grain de- formation. As the FWHM measure of the Laue spot will be utilized, it is necessary to ensure broadening arising from beam divergence is negligible compared to deformation in- duced smearing. Consequently, to achieve a practical focal spot of $10 \mathrm{~m}$, a divergence of $10-15$ mrad is considered adequate for the majority of applications.

To quantify the experimental broadening in the measured Laue data arising from beam divergence, it was necessary to collect a Laue pattern from a strain-free Si single crystal. Employing a diverging reflection geometry ${ }^{29}$ with the sample at the beam focus and image plate beyond the focus at a distance of $150 \mathrm{~mm}$, a measure of the divergence could be ascertained. A portion of the collected data is shown in Fig. 4. In this case the broadening associated with the measured Laue spot is the convolution of the incident beam spot size, $\mathrm{x}$-ray source energy distribution, beam divergence, and mo- 


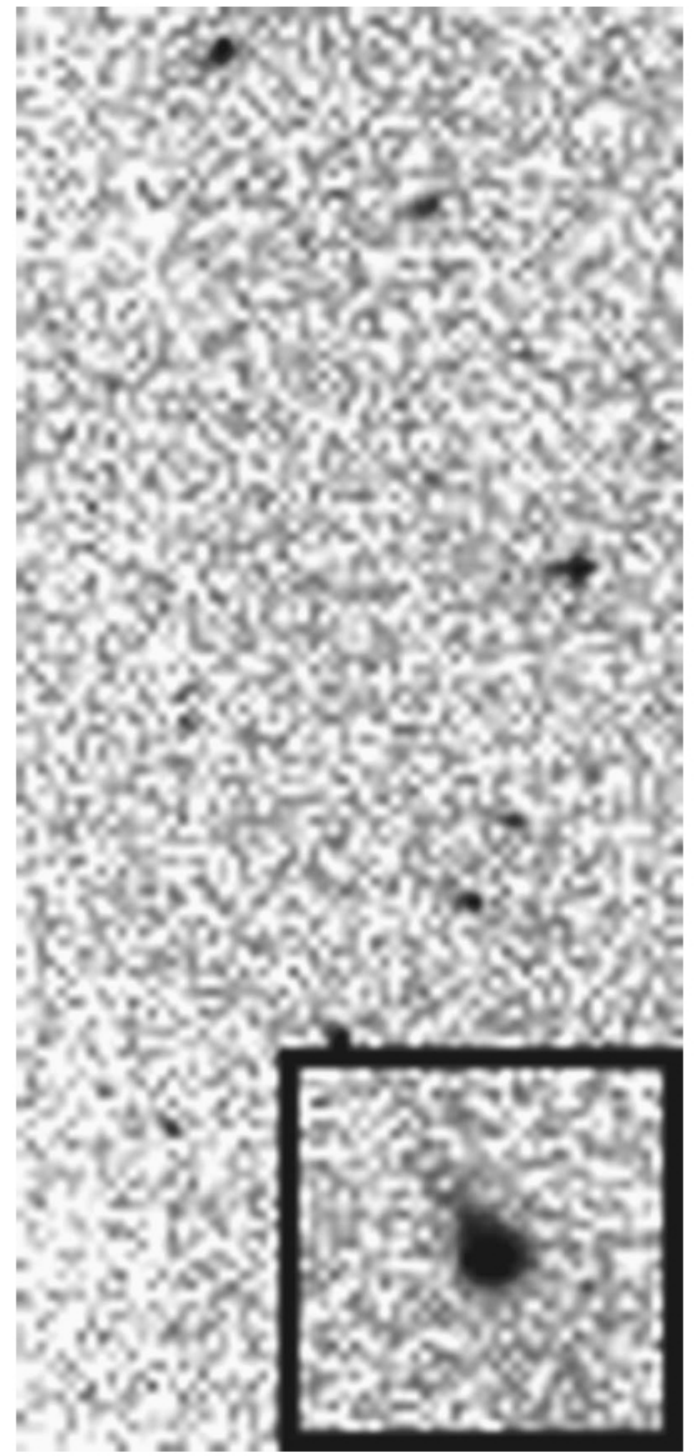

FIG. 4. Laue data from a Si 111 single crystal collected at a sample to image plate distance of $150 \mathrm{~mm}$, the inset shows a diffraction spot at 3 magnification.

saic spread of the crystal. Assuming divergence is the dominant effect, from measurement of the spot size and with knowledge of the image plate distance from the sample, the experimentally determined divergence was found to be 14 mrad which is in reasonable agreement with the theoretical divergence of $12 \mathrm{mrad}$.

For the present experimental setup the gain in $\mathrm{x}$-ray flux at the focal plane realized with the focusing capillary optic was measured. The optic performance was determined by recording the integrated intensity at the focal plane with the capillary in position, compared to the recorded integrated intensity for a $20 \mathrm{~m}$ aperture at the same distance from the source. Furthermore, to verify the measured capillary performance against the theoretically calculated gain for a single bounce ellipsoidal optic, several different source conditions were examined. Both the theoretical and experimentally measured optic gains are summarized in Table I.

The experimental results are in approximate agreement with theoretically calculated gain values for each source power loading. As an energy dispersive detector was not
TABLE I. Theoretical and experimental optic gain for a $20 \mathrm{~m}$ focused spot at four source power settings.

\begin{tabular}{cccc}
\hline \hline $\begin{array}{c}\text { Applied voltage } \\
\mathrm{kVp}\end{array}$ & $\begin{array}{c}\text { Applied current } \\
\mathrm{mA}\end{array}$ & $\frac{\text { Theoretical gain }}{\text { Experimental gain }}$ \\
30 & 1.00 & 700 & 507 \\
30 & 1.66 & 400 & 273 \\
30 & 2.33 & 270 & 176 \\
30 & 3.00 & 180 & 114 \\
\hline \hline
\end{tabular}

available to perform the gain measurements the difference between experimental and theoretical results may be due to the energy response of the image plates used to record the spot intensity. This is in agreement with experimental studies of an image plate pixel value response to the $\mathrm{x}$-ray beam energy spectrum. ${ }^{30}$ Furthermore, as a $\mathrm{x}$-ray shutter was not installed on the system a small error may arise in the experi- mental results due to slight timing discrepancies. Both the theoretical and experimental values highlight the impact of the source size power loading on the optic collection effi- ciency, i.e., as the power loading is increased the source size expands well beyond the $6 \mathrm{mrad}$ collection solid angle of the capillary entrance resulting in a substantial gain decrease. Finally, as the experimental gain is a maximum $73 \%$ at $30 \mathrm{kV}, 1 \mathrm{~mA}$ with respect to the theoretical result this is evi- dence the fabricated ellipsoidal optic shape is in close agree- ment with the initial theoretical design specifications based on a $30 \mathrm{~m}^{2} \mathrm{x}$-ray source.

\section{Energy spectrum}

The most challenging requirement of Laue diffraction on a laboratory level is to maximize the low-intensity continuum spectrum. In this instance, a x-ray tube with a high atomic number $Z$ anode material is seen as a considerable advantage, since the continuum radiation is proportional to $Z$. For example, due to the difference in atomic number between the elements $\mathrm{Cu}$ and $\mathrm{W}$, the observed white radiation from the $\mathrm{W}$ tube will be 2.5 more than a $\mathrm{Cu}$ tube operated at the same power. With this gain in intensity a $\mathrm{W}$ anode was essential for the present application. Another consideration in regard to the source was the applied power, as both the characteristic and continuum spectrum scale with applied power it was necessary to maximize this operating condition. However, as discussed in Sec. II the source size is also load dependent. Therefore, a compromise had to be made between the maximum intensity and minimum source size so the most effective collection efficiency of the optic could be realized. It was then necessary to test the ideal source settings with the optic in place. After several studies $30 \mathrm{kV}$ and 1-3 mA appeared to suit the optic/source combi-

nation, which equates to a source size of $30-90 \mathrm{~m}$.

The inherent characteristics of the capillary further assisted in obtaining a polychromatic bandpass for Laue diffraction experiments. That is, a focusing type capillary is designed with an incoming acceptance angle, within which incident photons of a given energy undergo total external reflection at the capillary walls and are focused at a point beyond the exit aperture. For the ellipsoidal capillary the acceptance angle was designed for an x-ray energy cutoff of 


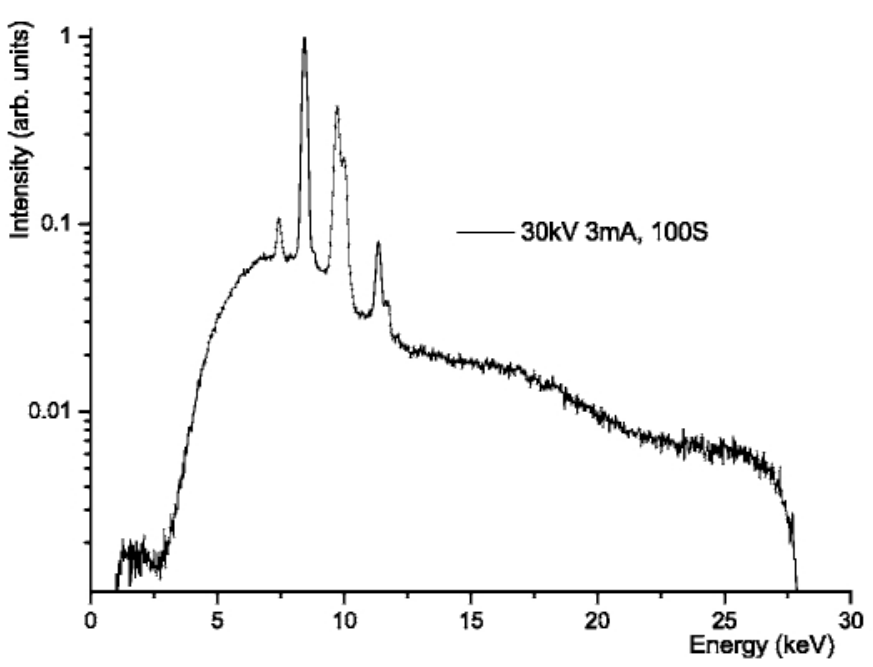

FIG. 5. Energy dispersive spectrum of the focused x-ray spot emerging from the capillary.

$22 \mathrm{keV}$. Therefore, ignoring efficiency losses, all x rays entering the capillary with an energy less than $22 \mathrm{keV}$ that undergo total external reflection will emanate from the capillary at the focus.

To measure the observed energy bandpass incident on the sample, an energy dispersive detector was placed $50 \mathrm{~mm}$ from the exit aperture of the capillary. The observed energy spectrum from the focused spot is given in Fig. 5. The recorded data was collected for $100 \mathrm{~s}$ with a source accelerat- ing voltage of $30 \mathrm{kV}$ and current of $3 \mathrm{~mA}$. Although the aim was to maximize the white radiation, due to the applied volt- age 30 $\mathrm{kV}$ the intensity of the characteristic $\mathrm{W} L$ and $\mathrm{W} L$ lines shown in Fig. 5 are significantly higher than the continuum maximum. From observation of the energy band- pass emanating from the capillary and from indexing the diffraction pattern arising from the Si reference material, outlined in Sec. II B, the useable energy bandpass of the capillary was from 5.5 to $19 \mathrm{keV}$.

It should be noted that the presence of characteristic and continuum components of the x-ray spectrum in the focused spot can be easily identified in the collected diffraction pattern. For example, when a specimen displays a random orientation with grain size equivalent and/or greater than the focused spot size, the probability of obtaining diffraction from a characteristic line is low and consequently the Laue patterns arise from the continuum spectrum. Conversely when a polycrystalline sample exhibits a grain size significantly smaller than the spot size a Debye Scherrer ring pat- tern is observed, since the probability of many small crystal- lites obeying the Bragg diffraction condition corresponding to the energy of the characteristic radiation is significantly increased. Therefore, the analysis procedure is highly dependent upon the specimen's grain size.

\section{B. Instrument calibration}

In terms of the instrumental requirements, one of the main prerequisites for crystal structure determination is pre- cise knowledge of the instrument geometry. Instrument cali- bration represents the first step toward the successful index-

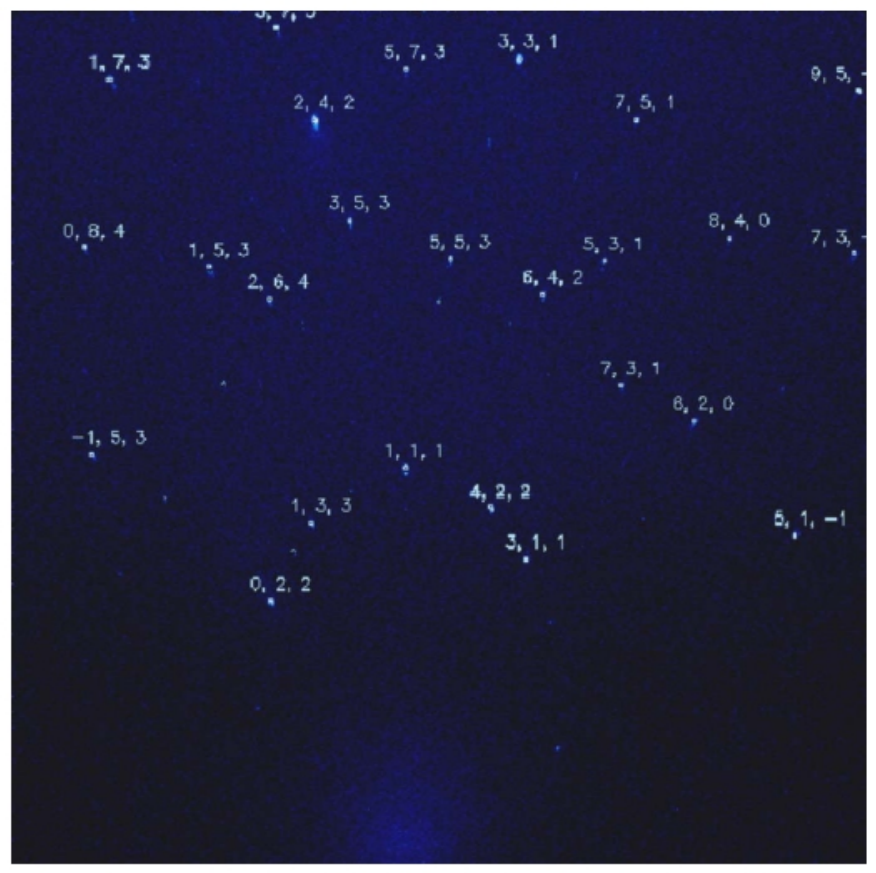

FIG. 6. Measured diffraction pattern from Si 111 single crystal reference material. Data collected at $30 \mathrm{kV}, 3 \mathrm{~mA}$ with an exposure time of $45 \mathrm{~min}$. White boxes and corresponding Miller indices represent the fit achieved for the experimental data.

ation of a measured Laue pattern and subsequent analysis. Calibration was performed by collecting data from an "ideal" strain free single crystal sample; for this task a Si 111 single crystal was used. The recorded reference pattern, given in Fig. 6, was obtained using a symmetric reflection geometry with the sample angle at $20^{\circ}$, image-plate angle 2 centered at $40^{\circ}$, at a distance of $45 \mathrm{~mm}$ from the sample. For these experiments image plates were preferred due to their high $\mathrm{x}$-ray sensitivity and the associated low intensity of the diffraction pattern. Diffraction patterns were also collected with a CCD detector and these preliminary CCD measurements will be discussed in Sec. IV.

For indexation of the Si single-crystal pattern and experimental patterns arising from the polycrystalline samples the XRD software package X-Ray Micro-Diffraction Analysis Software XMAS Refs. 31 and 32 was employed; for further details the reader is referred to Ref. 33. Prior to indexation, the known experimental conditions are required as input for the XMAS indexation package and these parameters include: the image-plate window size $100 \mathrm{~mm} 70 \mathrm{~mm}$; the and 2 angles of $20^{\circ}$ and $40^{\circ}$, respectively; energy bandpass of the tungsten source 5.5 to $19 \mathrm{keV}$; an estimate of the sample-to-detector distance $d d$

$45 \mathrm{~mm}$ and the center position of the image plate detector in the $x$ direction $X$ cent and $y$ direction Ycent , which were initially set to $X$ cent $=350$ and $Y$ cent $=500$.

After successfully indexing the Si reference pattern, further refinement of the geometrical parameters $d d$, Xcent , Ycent can be achieved by the method of triangulation. ${ }^{34}$ After recording several Si reference patterns by varying the distance between the specimen and image plate in $5 \mathrm{~mm}$ increments i.e., $d d=45,50, \ldots, 70 \mathrm{~mm}$ triangulation can be used to determine a more accurate measure of the distance 
TABLE II. Refined instrumental calibration parameters obtained from indexation of the Si reference material.

\begin{tabular}{ccccccc}
\hline \hline & 2 & & & $d d$ & $X$ & $X$ \\
$\circ$ & $2^{2}$ & $\underline{X}_{\text {cent }}$ & $\underline{Y}_{\text {cent }}$ & $\underline{\mathrm{mm}}$ & $\circ$ & $\circ$ \\
- & 40 & 920.5 & 730.5 & 46 & -0.75 & -0.2 \\
20 & & & & & & \\
\hline \hline
\end{tabular}

$d d$ for the closest recorded pattern $d d=45 \mathrm{~mm}$ together with the $X$ cent and $Y$ cent values for this pattern. After updat- ing these parameters, final calibration of the instrumental parameters including the detector tilt and rotation angles Xalfd, Xbetd, and Xgam can be refined for the reference material. These final calibrated parameters are listed in Table II. The final indexation result for the Si reference pattern, obtained with these settings, is illustrated in Fig. 6. Indexed Laue spots in the collected data are signaled by a white box with associated Miller indices. Ensuring the instrument geometry is unchanged the geometric settings given in Table II are incorporated as instrument input parameters for indexation of samples with unknown orientation.

\section{OBSERVATION OF GRAIN ORIENTATION AND LATTICE STRAIN IN MgNd}

To highlight the system capability to track the orientation and strain of individual grains in a polycrystalline material, diffraction measurements were collected from a cast MgNd 99.98\% Mg, 0.2\% Nd alloy sample. The cast Mg alloy was prepared by hot rolling to a thickness of $1 \mathrm{~mm}$. After a post-rolling anneal the sample was polished to pro- vide an estimate of the average grain size Fig. 7 . From optical microscopy measurements grains were found to be approximately equiaxed with a size varying from $10 \mathrm{~m}$ to $100 \mathrm{~m}$.

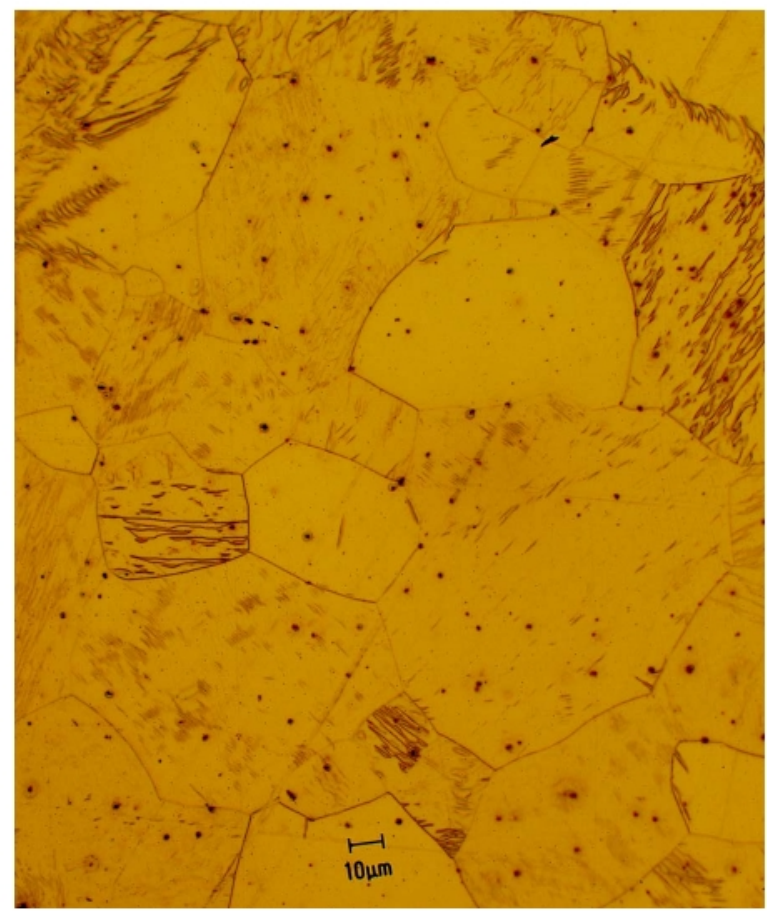

FIG. 7. Optical microscope image of the polished Mg-Nd sample providing an indication of the average grain size, magnification 50 .

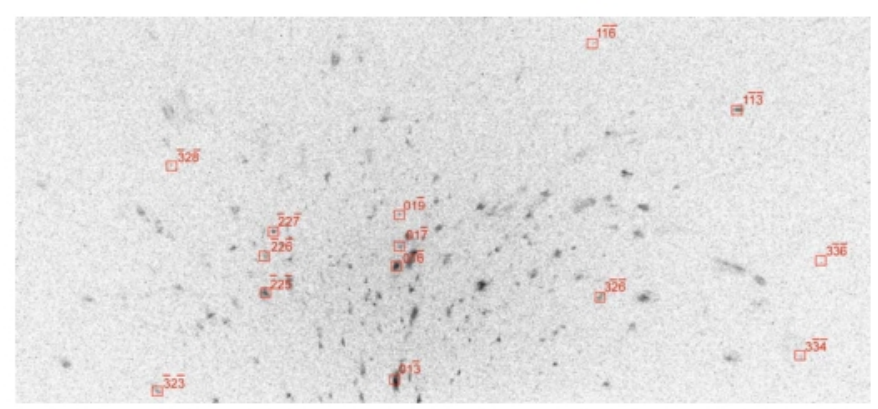

FIG. 8. Experimental diffraction pattern from the annealed Mg-Nd sample, exposure time $1 \mathrm{~h}$. Squares and Miller indices represent indexation of the "'highest integrated intensity" grain.

For the present study two sets of XRD measurements were collected; the first set was recorded for the annealed sample while the second set was collected after the specimen had undergone uniaxial tensile loading to an engineering strain of $2.5 \%$. All data collection was undertaken using the same experimental geometry as employed for the Si reference material. That is, symmetric reflection with the sample angle $=20^{\circ}, 2=40^{\circ}$ and the refined sample-to-detector distance $d d=46 \mathrm{~mm}$. To provide a measure of the change in orientation and strain of neighboring grains the scanning

XRD approach was used. ${ }^{35}$ In this mode, the sample is raster scanned $20 \mathrm{~m}$ steps under the focused $\mathrm{x}$-ray beam. For each step increment a diffraction pattern was recorded, in total a $100 \mathrm{~m}$ line scan was collected before and after ten- sile loading. For the $\mathrm{W} x$-ray energy spectrum emanating from the capillary optic, at $10 \mathrm{keV}$ the attenuation length of $\mathrm{Mg}$ is 300 m. As a result it was anticipated that each recorded diffraction pattern would represent an illumination of 10-15 grains. An example of the recorded data for the annealed sample is given in Fig. 8. From the complex "spot- ted" diffraction pattern it is clearly evident that the grain size is greater than or of similar size to the incident beam size.

Due to the complexity of the measured patterns only grains indexed with at least 15 diffraction spots were considered. Based on this criteria it was possible to determine the orientation matrix of about ten grains in each experimental pattern. It should be noted with image-plate data collection, time requirements made it prohibitive to perform triangula- tion to resolve depth information of the diffracting grains. It is anticipated the efficiency of this aspect of the work will be greatly improved by incorporating a CCD detector, an area of future development for the present system. Consequently, the results presented are based on the indexed grain from each image which displays the highest integrated intensity. For example, indexation of the diffraction spots from a single grain, each spot highlighted by a white box with associated Miller indices, for the annealed specimen is illustrated in Fig. 8.

Utilizing the refined orientation matrices for each of the "highest integrated intensity" grains, the out-of-plane and in- plane orientations were calculated. Results for the line scan data are illustrated in Fig. 9 for the Mg-Nd sample before and after tensile loading. Figures 9 a and 9 c show the relative change in the angle between the 0002 direction and surface normal for each grain before and after tensile loading 

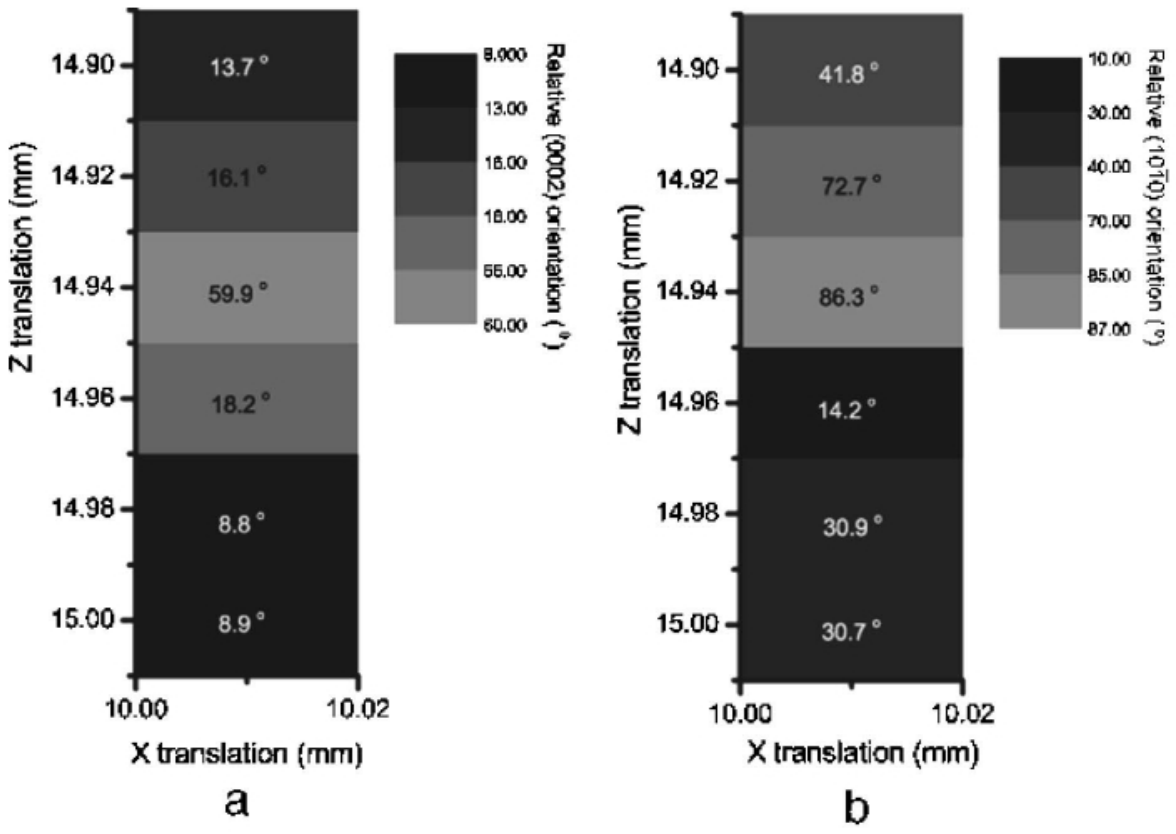

FIG. 9. Out-of-plane and in-plane tensile axis orientation from indexed grains that exhibit the highest integrated intensity from the line scan data of the annealed a , b and tensile deformed c , d Mg-Nd sample. a , c Out-of-plane orientation relative to 0002 , b , d In-plane orientation relative to $101 \overline{0}$, parallel to the tensile axis.
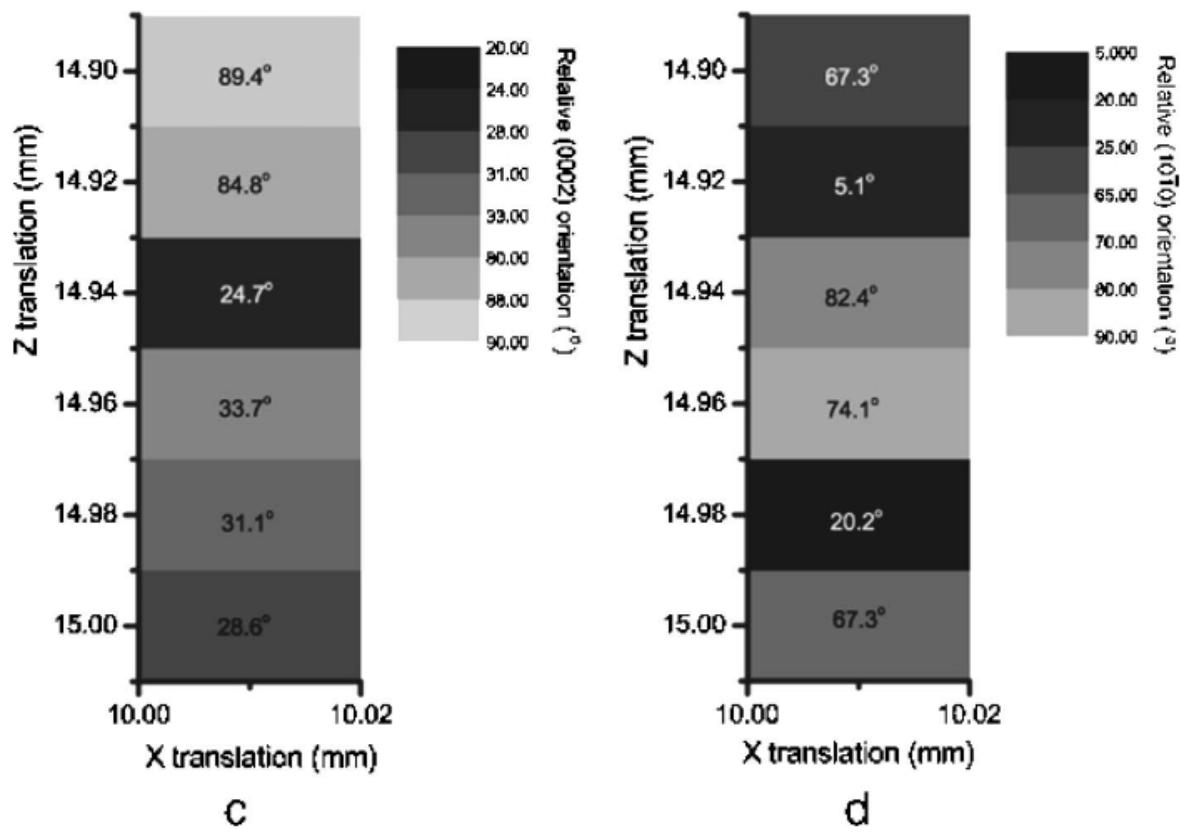

respectively. Figures $9 \mathrm{~b}$ and $9 \mathrm{~d}$ show the relative change

in the angle between the $10 \overline{1} 0$ direction and the in-plane sample $x$ direction tensile axis for each grain before and after tensile loading, respectively. In the annealed state the majority of Mg-Nd grains display a basal orientation with a random in-plane orientation, a texture commonly encountered in bulk measurement studies from rolled Mg alloys. ${ }^{36,37}$ An indication of the grain size can also be ascertained from the sample scanning approach. For the $Z$ stage positions of 14.98 and $15.00 \mathrm{~mm}$ the out-of-plane and in-plane orienta- tions are practically equal Figs. 9 a and $9 \mathrm{~b}$. This signifies diffraction from the same grain at least $40 \mathrm{~m}$ in size. In principle, the resolution of the grain size measurement is dictated by the step size.

For the loaded sample some of the grains in the initial basal texture appear to undergo an orientation change of $30^{\circ}$ with respect to 0002 , initiated by plastic deformation at a load of $2.5 \%$ strain. Since the sample had to be removed to perform tensile loading it was not possible to locate the same grain set to make a direct comparison, hence relative changes discussed in this study are restricted to grains from a similar region in the sample. Independent of this, further studies are presently being undertaken to determine the microstructural significance of the observed orientation change. In conjunc- tion with the observed orientation change the induced plastic deformation also produced streaking broadening and split- ting of the diffraction spots. These effects are caused by dis- location slip promoting grain sub-division. This is in agree- ment with the collected line scans showing unique grains for each step increment. This aspect of the work, determining the twin type and/or slip mechanisms is currently in progress.

An additional feature of the diffraction measurements is the ability to measure the induced lattice strain d-spacing 


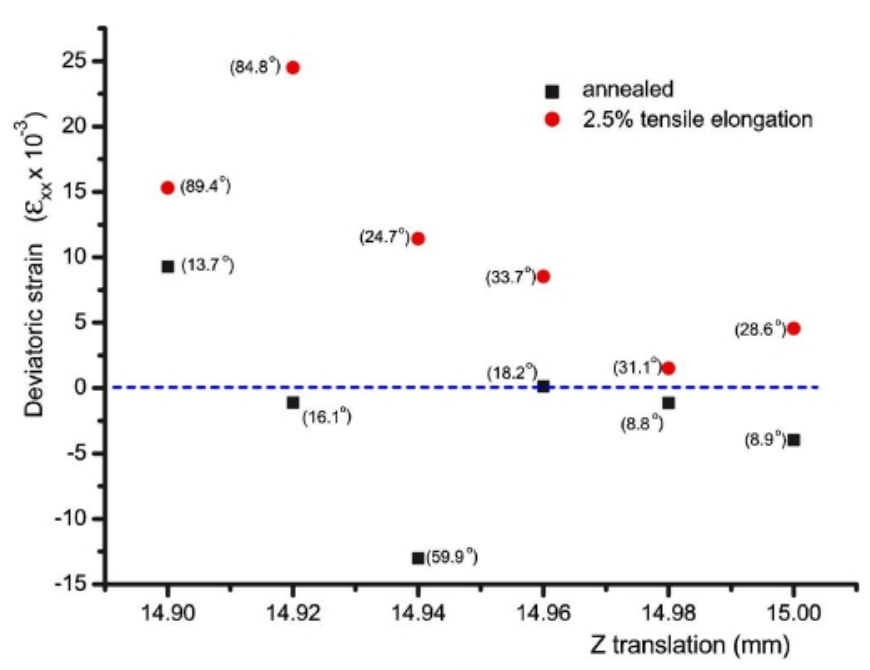

a

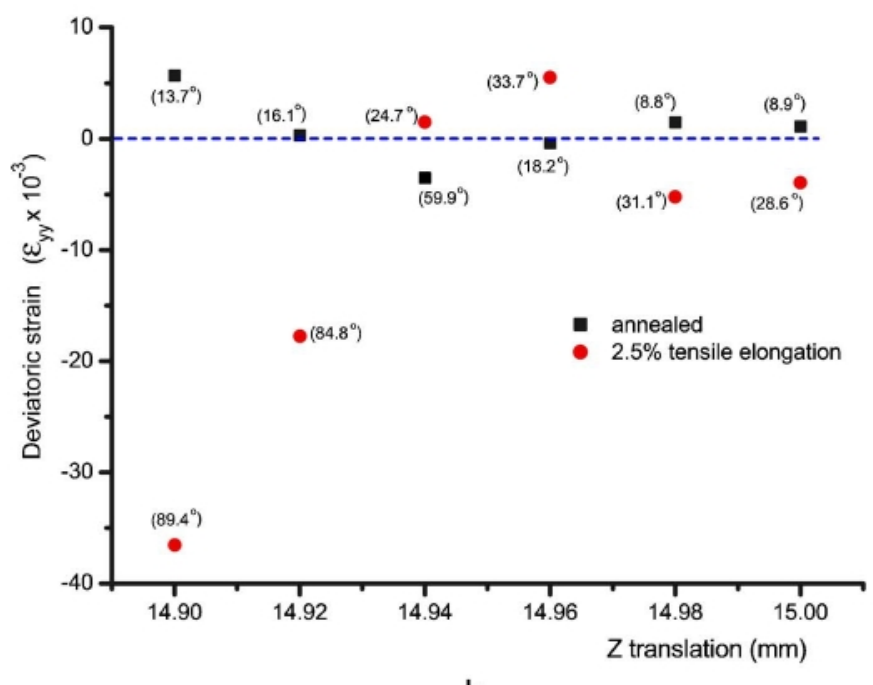

b

FIG. 10. Relative in-plane strain observed in the highest integrated intensity grains before and after an applied strain of $2.5 \%$. a Normal strain compo- nent parallel to the elongation axis. b Normal strain component perpen- dicular to the elongation axis. Values in brackets indicate out-of-plane orientation with respect to 0002 .

based on the observed spot positions relative to the "strain free" state. Expressed in tensor notation the measured strain can be specified for a given sample direction. The results for the inplane strain components $X X$ and ${ }_{Y Y}$ for the line scan data are illustrated in Fig. 10. As mentioned, it was not possible to make a direct comparison between grains before and after loading and so relative lattice strain shifts are based on an average effect. Prior to deformation, the points for the in-plane strain, in the tensile direction ${ }_{X X}$ and transverse direction ${ }_{Y Y}$ are approximately zero, a result consistent with the post rolling anneal. After tensile loading, the majority of grains exhibit a tensile strain parallel to the load axis and compressive component perpendicular to the tensile axis.

\section{DISCUSSION}

As demonstrated for the MgNd alloy, the microdiffraction system and subsequent data processing was used to determine the orientation matrix and strain tensor of individual grains in a bulk matrix before and after load application. The ability to measure this microstructural information at an individual grain level should provide useful input and verifica- tion for the next generation of deformation models. For ex- ample, the ability to track the orientation of individual grains provides a precise method for determining twinning mecha- nisms as a function of applied load. Moreover, the ability to quantify individual grain structure information from a labo- ratory instrument serves as a highly useful preliminary data set if further high-resolution XRD studies are to be per-

formed at a synchrotron facility.

To improve the statistical relevance of the measured individual grain data, an increased population of grains must be characterized. This is an area which demands further instrument development. Having proven that the weakly scattering diffraction pattern can be adequately collected using image plates, a primary focus of continued instrument development lies in the area of data collection automation. For the samples studied in this report preliminary measurements have been carried out with an uncooled CCD detector. Even with the poor dark current characteristics of the present CCD it was possible to collect diffraction patterns within

30 min. Further work is planned using a cooled CCD with an increased active area size. The addition of data collection automation will greatly assist in the ability to track a greater ensemble of grains and will also improve triangulation methods to establish the scattering depth of individual grains.

\section{CONCLUSION}

By utilizing the small spot size capabilities of a microfocus $x$-ray source coupled with the flux gain achieved with a single bounce capillary optic, a laboratory microdiffraction system with a spatial resolution of $10 \mathrm{~m}$ was realized. In conjunction with the high-spatial resolution, the continuous nature of the x-ray polychromatic spectrum of the tungsten source enabled diffraction measurements to be performed without requiring sample rotation. Indexation of the experimental diffraction pattern arising from individual grains in a bulk polycrystalline Mg-Nd alloy was achieved with the software package XMAS. Using a raster scan approach and with subsequent analysis, for the majority of grains, a gen- eral basal 0002 grain orientation was observed in the post roll annealed Mg-Nd sample. After tensile loading to an engineering strain of $2.5 \%$, the individual grain texture appeared to undergo an orientation change of $30^{\circ}$ with respect to 0002 . The applied strain was quantified as an in-plane tensile and compressive strain parallel and perpendicular to the load axis respectively.

\section{ACKNOWLEDGMENTS}

The authors would like to thank K. Venkatesan for preparing and performing the strain test on the MgNd sample, C. Bettles and M. Gibson for scientific discussions and encouragement throughout the development of this project. This work was supported by the Victorian Centre for Advanced Materials Manufacturing VCAMM .

${ }^{1}$ G. C. Butler, S. R. Stock, R. D. McGinty, and D. L. McDowell, J. Eng. Mater. Technol. 124, 482002.

2 J. S. Chung and G. E. Ice, J. Appl. Phys. 86, 52491999 . 
${ }^{3}$ R. Barabash, G. E. Ice, B. C. Larson, G. M. Pharr, K.-S. Chung, and W. Yang, Appl. Phys. Lett. 79, 7492001.

${ }^{4}$ R. I. Barabash, G. E. Ice, B. C. Larson, and W. Yang, Rev. Sci. Instrum. 73, 16522002 .

${ }^{5}$ O. Hignette, P. Cloetens, G. Rostaing, P. Bernard, and C. Morawe, Rev. Sci. Instrum. 76, 0637092005.

${ }^{6}$ M. R. Barnett, M. D. Nave, and C. J. Bettles, Mater. Sci. Eng., A 386, 205 2004.

${ }^{7}$ B. A. GMBH Technical Report Bruker AXS, Inc., 1998.

${ }^{8}$ P. Kirkpatrick and A. V. Baez, J. Opt. Soc. Am. 38, 7561948.

${ }^{9}$ E. D. Fabrizio, F. Ramanato, M. Gentili, S. Cabrini, B. Kaulich, J. Susini, and R. Barnett, Nature London 9, 895-898 1999 .

${ }^{10}$ M. Altissimo, F. Romanato, L. Vaccari, L. Businaro, D. Cojoc, B. Kaulich, S. Cabrini, and E. D. Fabrizio, Microelectron. Eng. 61-62, 1732002 .

${ }^{11}$ D. H. Bilderback, S. A. Hoffman, and D. J. Thiel, Science 263, 201 1994.

${ }^{12}$ S. Wilkins, A. W. Stevenson, K. A. Nugent, H. Chapman, and S. Steenstrup, Rev. Sci. Instrum. 60, 10261989

${ }^{13}$ A. Snigirev, V. Kohn, I. Snigirev, and B. Lengeler, Nature 384, 491996. ${ }^{14}$ D. H. Bilderback, X-Ray Spectrom. 32, 1952003.

${ }^{15}$ D. X. Balaic, K. Nugent, Z. Barnea, R. Garrett,and S. Wilkins, J. Synchrotron Radiat. 2, 2961995.

${ }^{16}$ A. H. Compton and S. K. Allison, X-Rrays inTheory and Experiment Van Nostrand, New York, 1935.

${ }_{17}$ A. Rindby, Nucl. Instrum. Methods Phys. Res. A 249, 5361986.

${ }^{18}$ D. A. Carpenter, X-Ray Spectrom. 18, 2531989.

${ }^{19}$ D. J. Thiel, D. H. Bilderback, A. Lewis, E. A. Stern, and T. Rich, Appl. Opt. 31, 9871992 .

${ }^{20}$ P. Engstrom, S. Larsson, A. Rindby, A. Buttkewitz, S. Garbe, G. Gaul, A. Knochel, and F. Lechtenberg, Nucl. Instrum. Methods Phys. Res. A 302, 5471991 .

${ }^{21}$ N. Yamamoto and Y. Hosokawa, Jpn. J. Appl. Phys., Part 2 27, L2203 1988.
${ }^{22}$ A. Attaelmanan, P. Voglis, A. Rindby, S. Larsson, and P. Engstrom, Rev. Sci. Instrum. 66, 241995.

${ }^{23}$ D. X. Balaic and K. A. Nugent, Appl. Opt. 34, 72631995.

${ }^{24}$ N. Yamamoto, Rev. Sci. Instrum. 67, 30511996

${ }^{25}$ D. WJgrzynek, X-Ray Spectrom. 30, 4132001.

${ }^{26}$ A. Bjeoumikhov, N. Langhoff, J. Rabe, and R. Wedell, X-Ray Spectrom.

33, 3122004.

${ }^{27}$ D. E. Grider, A. Wright, and P. K. Ausburn, J. Phys. D 19, 22811986.

${ }^{28}$ D. X. Balaic, Australian X-ray Capillary Optics Pty Ltd. AXCO . http://

axco.com.au

${ }^{29}$ D. X. Balaic, Z. Barnea, K. A. Nugent, R. F. Garrett, J. N. Varghese, and

S. Wilkins, J. Synchrotron Radiat. 3, 2891996.

${ }^{30}$ D. M. Tucker, P. S. Rezentes, and A. Assoc, Phys. Med. 24, 8871997.

${ }^{31}$ N. Tamura, A. A. MacDowell, R. Spolenak, B. C. Valek, J. C. Bravman,

W. L. Brown, R. S. Celestre, H. A. Padmore, B. W. Batterman, and J. R. Patel, J. Synchrotron Radiat. 10, 1372003.

32 XMAS, X-ray microdiffraction analysis software, is a software suite designed specifically for the analysis of $\mathrm{x}$-ray microdiffraction data collected on beamline 7.3.3 at the Advanced Light Source ALS . The software can be freely downloaded on the ALS microdiffraction website http:// xraysweb.lbl.gov/microdif/XMAS/

${ }_{33}$ XMAs indexing software tutorial v.1. This tutorial is available for free download from the ALS microdiffraction website http://xraysweb.lbl.gov/ microdif/XMAS/

${ }^{34}$ B. C. Larson, N. Tamura, J. S. Chung, G. E. Ice, J. D. Budai, J. Z. Tischler, W. Yang, H. Weiland, and W. P. Lowe, Mater. Res. Soc. Symp. Proc. 590, 2472000

${ }^{35}$ N. Tamura, H. Padmore, and J. R. Patel, Mater. Sci. Eng., A 399, 92 2005.

${ }^{36}$ Magnesium Alloys and Their Applications, edited by K. U. Kainer WileyVCH, Weinheim, pp. 119-124.

${ }^{37}$ F. Kaiser, J. Bohlen, D. Letzig, K.-U. Kainer, A. Styczynski, and C. Hartig, Adv. Eng. Mater. 5, 8912003. 